\title{
Growth and Pattern Formation for Thin Films
}

\author{
Russel E. Caflisch \\ Department of Mathematics and Department of Material Science \& Engineering, \\ University of California, 405 Hilgard Avenue, Los Angeles, CA 90095-1555, USA \\ caflisch@math.ucla.edu
}

Summary. Epitaxy is the growth of a thin film by attachment to an existing substrate in which the crystalline properties of the film are determined by those of the substrate. In heteroepitaxy, the substrate and film are of different materials, and the resulting mismatch between lattice constants can introduce stress into the system. We have developed an island dynamics model for epitaxial growth that is solved using a level set method. This model uses both atomistic and continuum scaling, since it includes island boundaries that are of atomistic height, but describes these boundaries as smooth curves. The strain in the system is computed using an atomistic strain model that is solved using an algebraic multigrid method and an artificial boundary condition. Using the growth model together with the strain model, we simulate pattern formation on an epitaxial surface.

\section{Introduction}

Epitaxy is the growth of a thin film on a substrate in which the crystal properties of the film are inherited from those of the substrate. Since an epitaxial film can (at least in principle) grow as a single crystal without grain boundaries or other defects, this method produces crystals of the highest quality.

The geometry of an epitaxial surface consists of step edges and island boundaries, across which the height of the surface increases by one crystal layer, and adatoms which are weakly bound to the surface. Epitaxial growth involves deposition, diffusion, and attachment of adatoms on the surface. Deposition is from an external source, such as a molecular beam. The principal dimensionless parameter (for growth at low temperature) is the ratio $D /\left(a^{4} F\right)$, in which $a$ is the lattice constant and $D$ and $F$ are the adatom diffusion coefficient and deposition flux. It is conventional to refer to this parameter as $D / F$, with the understanding that the lattice constant serves as the unit of length. Typical values for $D / F$ are in the range of $10^{4}-10^{8}$. 


\section{Island Dynamics}

Burton, Cabrera, and Frank [2] developed the first detailed theoretical description for epitaxial growth. In this "BCF" model, the adatom density solves a diffusion equation with an equilibrium boundary condition $\left(\rho=\rho_{\text {eq }}\right)$, and step edges (or island boundaries) move at a velocity determined from the diffusive flux to the boundary. Modifications of this theory were made, for example in [11], to include line tension, edge diffusion, and nonequilibrium effects. These are "island dynamics" models, since they describe an epitaxial surface by the location and evolution of the island boundaries and step edges. They employ a mixture of coarse graining and atomistic discreteness, since island boundaries are represented as smooth curves that signify an atomistic change in crystal height.

Adatom diffusion on the epitaxial surface is described by a diffusion equation of the form

$$
\partial_{\mathrm{t}} \rho-D \nabla^{2} \rho=F-2 \mathrm{~d} N_{\text {nuc }} / \mathrm{d} t
$$

in which the last term represents loss of adatoms due to nucleation, and desorption from the epitaxial surface has been neglected. Attachment of adatoms to the step edges and the resulting motion of the step edges are described by boundary conditions at an island boundary (or step edge) $\Gamma$ for the diffusion equation and a formula for the step-edge velocity $v$. The simplest of these is

$$
\begin{aligned}
\rho & =\rho_{*} \\
v & =D[\partial \rho / \partial n]
\end{aligned}
$$

in which the brackets indicate the difference between the value on the upper side of the boundary and the lower side. Two choices for $\rho_{*}$ are $\rho_{*}=0$, which corresponds to irreversible aggregation in which all adatoms that hit the boundary stick to it irreversibly, and $\rho_{*}=\rho_{\text {eq }}$ for reversible aggregation. For the latter case, $\rho_{\mathrm{eq}}$ is the adatom density for which there is local equilibrium between the step and the terrace [2]. Numerical details on implementation of the level set method for thin film growth are provided in [5].

\subsection{Nucleation}

For the case of irreversible aggregation, a dimer (consisting of two atoms) is the smallest stable island, and the nucleation rate is

$$
\frac{\mathrm{d} N_{\text {nuc }}}{\mathrm{d} t}=D \sigma_{1}\left\langle\rho^{2}\right\rangle,
$$

where $\langle\cdot\rangle$ denotes the spatial average of $\rho(\mathbf{x}, t)^{2}$ and

$$
\sigma_{1}=\frac{4 \pi}{\ln [(1 / \alpha)\langle\rho\rangle D / F]}
$$


is the adatom capture number as derived in [1]. The parameter $\alpha$ reflects the island shape, and $\alpha \simeq 1$ for compact islands. Expression (3) for the nucleation rate implies that the time of a nucleation event is chosen deterministically. Whenever $N_{\text {nuc }} L^{2}$ passes the next integer value ( $L$ is the system size), a new island is nucleated. Numerically, this is realized by raising the level set function to the next level at a number of grid points chosen to represent a dimer.

The choice of the location of the new island is determined by probabilistic choice with spatial density proportional to the nucleation rate $\rho^{2}$. This probabilistic choice constitutes an atomistic fluctuation that must be retained in the level set model for faithful simulation of the epitaxial morphology. For growth with compact islands, computational tests have shown additional atomistic fluctuations can be omitted [16].

Additions to the basic level set method, such as finite lattice constant effects and edge diffusion, are easily included [17]. The level set method with these corrections is in excellent agreement with the results of kinetic Monte Carlo (KMC) simulations.

\subsection{The Level Set Method}

Within the level set approach, the union of all boundaries of islands of height $k+1$, can be represented by the level set $\varphi=k$, for each $k$. For example, the boundaries of islands in the submonolayer regime then correspond to the set of curves $\varphi=0$. The function $\phi$ is the level set function that evolves according to

$$
\frac{\partial \phi}{\partial t}+v|\nabla \phi|=0 .
$$

All the physical information is in the normal component $v$ of the velocity function. Islands grow because atoms diffuse toward and attach to island boundaries, and shrink because they can detach from an island boundary.

\section{Discrete Elasticity}

In heteroepitaxy, strain is introduced into the epitaxial system due to the lattice mismatch between the two constituents of the material. Because of the strain, atoms are displaced by a vector $\mathbf{u}$ from their lattice position. The following discussion of atomistic strain and stress follows that in [19].

To describe the strain energy at each atom, $\mathbf{i}=(i, j, k)$, introduce the translation operators, $T_{k}^{ \pm}$, and the discrete difference operators, $D_{k}^{ \pm}, D_{k}^{0}$, defined as follows:

$$
\begin{aligned}
& T_{k}^{ \pm} f(\mathbf{i})=f\left(\mathbf{i} \pm \mathbf{e}_{\mathbf{k}}\right), \\
& D_{k}^{+} f(\mathbf{i})=\frac{\left(T_{k}^{+}-1\right) f(\mathbf{i})}{h},
\end{aligned}
$$




$$
\begin{aligned}
D_{k}^{-} f(\mathbf{i}) & =\frac{\left(1-T_{k}^{-}\right) f(\mathbf{i})}{h}, \\
D_{k}^{0} f(\mathbf{i}) & =\frac{\left(T_{k}^{+}-T_{k}^{-}\right) f(\mathbf{i})}{2 h},
\end{aligned}
$$

where $h$ is the lattice constant and $\mathbf{e}_{k}$ is the vector in the $k$ th direction for $k=$ $1,2,3$ with $\left\|\mathbf{e}_{k}\right\|=h$. Throughout this paper, we assume the lattice constant $h=1$ for simplicity. We use $i$ for the depth-like index, with $-\infty<i \leq n$. Here $n$ is the maximum height of the material. An ABC is sought at $i=0$, assuming that there is no force for $i<0$.

Let $\mathbf{u}(\mathbf{i})=\left(u_{k}(\mathbf{i})\right)_{k=1, \ldots, d}$ be the displacement at the discrete point $\mathbf{i}$ relative to an equilibrium lattice. The discrete strain components defined below $((6)$ and $(7))$ can be used to describe the discrete elastic energy. For $k, \ell=1,2,3$ and $p, q= \pm$,

$$
\begin{aligned}
S_{k \ell}^{ \pm}(\mathbf{u}(\mathbf{i})) & =D_{\ell}^{ \pm} u_{k}(\mathbf{i}), \\
S_{k \ell}^{p q}(\mathbf{u}(\mathbf{i})) & =\frac{1}{2}\left(D_{\ell}^{q} u_{k}(\mathbf{i})+D_{k}^{p} u_{\ell}(\mathbf{i})\right) .
\end{aligned}
$$

The discrete energy density at a point $\mathbf{i}$ is then given by

$$
E(\mathbf{i})(\mathbf{u}, \mathbf{u})=\sum_{k, p} \alpha_{k}^{p}\left(S_{k k}^{p}(\mathbf{u})\right)^{2}+\sum_{k \neq \ell, p, q}\left\{2 \beta_{k \ell}^{p q}\left(S_{k \ell}^{p q}(\mathbf{u})\right)^{2}+\gamma_{k \ell}^{p q} S_{k k}^{p}(\mathbf{u}) S_{\ell \ell}^{q}(\mathbf{u})\right\} .
$$

The total energy is the sum

$$
\mathcal{E}=\sum_{\mathbf{i}} E(\mathbf{i})
$$

The atomistic strain is determined by minimizing this energy with respect to variations in $\mathbf{u}$.

An effective numerical method for solving the atomistic strain equations using an algebraic multigrid method was developed in [4]. Moreover an artificial boundary condition can be imposed in the substrate close to the interface with the film, to greatly accelerate the computation [10].

\section{Directed Self-Assembly}

Regular patterns of nanoscale features, such as quantum dots $[6,7,12]$, on an epitaxial surface are of considerable interest for possible applications, ranging from memory and logical devices to lasers. Features of this size are difficult to obtain by standard "top-down" approaches, such as lithography. The spontaneous growth of quantum dot arrays is a promising "bottom-up" approach, but it has proved difficult to control the size and spacing of quantum dots obtained in this way. Directed self-assembly is an intermediate approach, in 
which formation of the desired patterns is guided by prepatterning of the epitaxial system. For example, subsurface dislocation arrays have been suggested as a prepatterning method $[8,18]$. These buried dislocations introduce a long-range strain field, which alters the potential energy surface (PES) of the system. Similarly, islands that are capped by a buffer layer of a different material introduce a long-range strain field. It has been shown by densityfunctional theory (DFT) calculations for metal systems [15] and semiconductor systems [14] that both the adsorption energy $E_{\text {ad }}$ and the transition energy $E_{\text {trans }}$ of the PES change upon strain.

We model epitaxial growth on a surface with a spatially varying, anisotropic $\mathrm{PES}$, using the following modification of the adatom diffusion equation (1)

$$
\frac{\partial \rho}{\partial t}=F+\nabla \cdot(\mathbf{D} \nabla \rho)-2 \frac{\mathrm{d} N}{\mathrm{~d} t}+\nabla \cdot\left(\frac{\rho}{k_{B} T} \mathbf{D}\left(\nabla E_{\mathrm{ad}}\right)\right) .
$$

In (9), $\mathbf{D}$ is a diffusion tensor where the diagonal entries are labeled $D_{i}(\mathbf{x})$ and $D_{j}(\mathbf{x})$, and correspond to diffusion along the two directions $i$ and $j$. For simplicity no other direction for diffusion is included (but could easily be incorporated). The last term is the thermodynamic drift, where $k_{\mathrm{B}}$ is the Boltzmann constant, and $T$ is the temperature. We enforce a boundary condition $\rho(\mathbf{x})=\rho_{\text {eq }}\left(D_{\text {det }}(\mathbf{x}), \mathbf{x}\right)$, where $D_{\text {det }}(\mathbf{x})$ is a (spatially varying) detachment rate $[3]$.

We assume a simple sinusoidal variation of $E_{\text {ad }}$ and $E_{\text {trans }}$. Figure 1 shows the resulting patterns for PES with spatial variation that is one dimensional (left) and two dimensional (right). These simulation results bear a striking resemblance to the quantum dot patterns obtained in the experimental results of $[8]$.

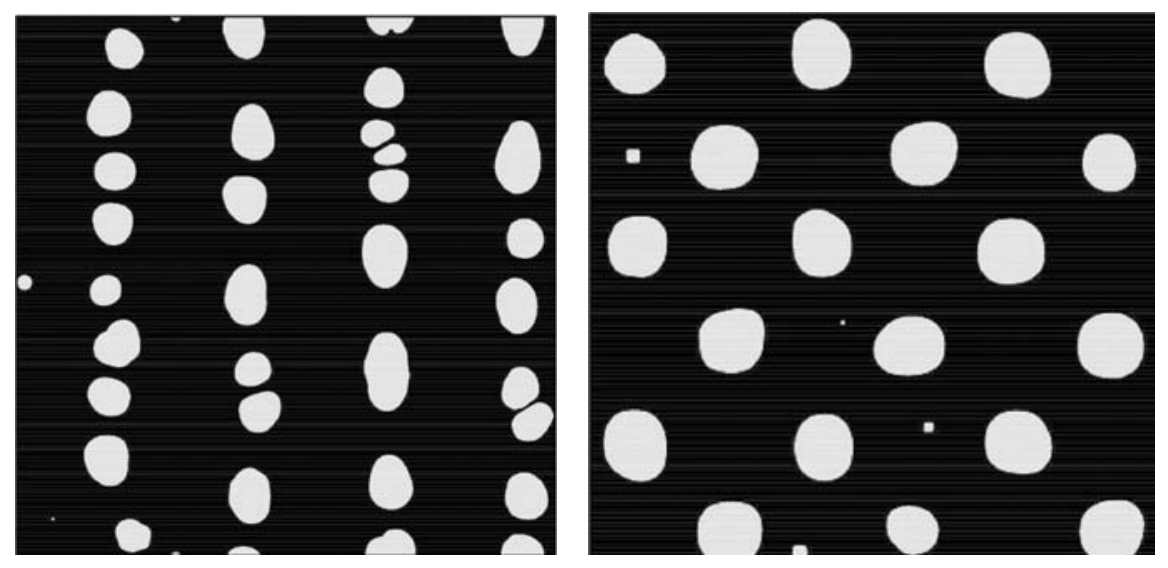

Fig. 1. Pattern formation for monolayer height islands due to a spatially varying $\mathrm{PES}$, with sinusoidal variation in $1 \mathrm{D}$ (left) and $2 \mathrm{D}$ (right) 

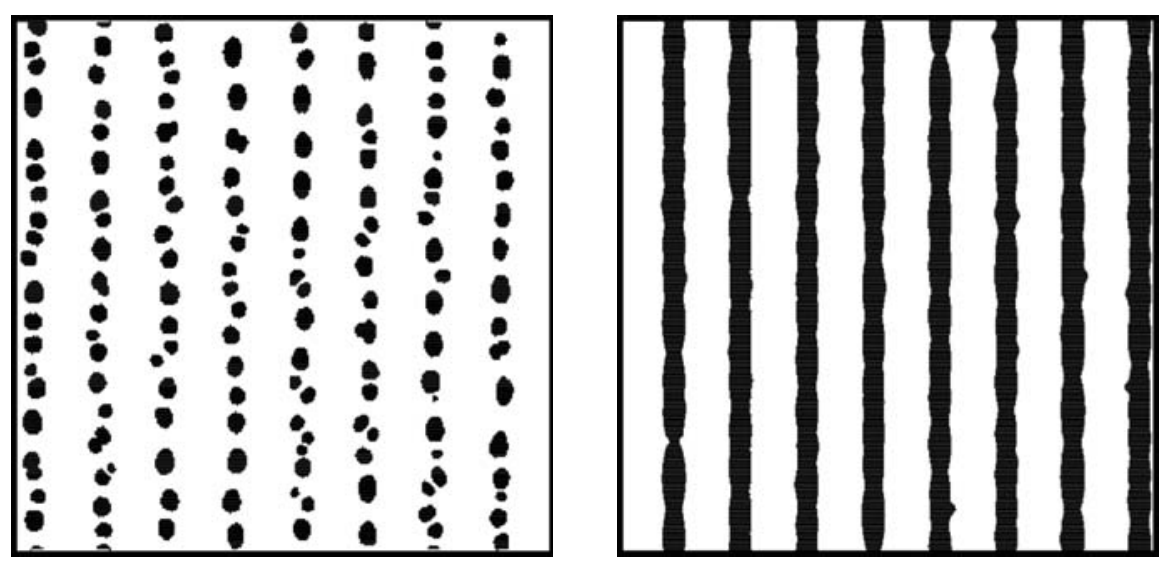

Fig. 2. Morphologies at coverages $\Theta=0.1 \mathrm{ML}$ (left) and $\Theta=0.3 \mathrm{ML}$ (right) obtained with a PES that has a much narrower variation

The morphologies shown so far were all obtained at a submonolayer precoalescence coverage of $\Theta=0.2 \mathrm{ML}$ and with a PES that varies sinusoidally. Figure 2 shows the patterns that are obtained by a function that has sharper peaks that those of a sine function. The resulting islands at coverage $\Theta=0.1$ monolayer (ML) are highly aligned. Moreover, at $\Theta=0.3 \mathrm{ML}$, all the islands that are aligned along the $j$-direction have coalesced in this direction, forming monolayer height "wires." For more details on these computations, see [13].

\section{Conclusions}

The island dynamics/level set method is capable of simulating epitaxial growth with processes such as adatom detachment from islands that would slow down other approaches. It can also be effectively combined with an atomistic strain code to simulate heteroepitaxial growth. The combined method can be used to study pattern formation due to strain in self-assembly and directed self-assembly.

\section{Acknowledgements}

This research was supported in part by the MARCO Center on Functional Engineered NanoArchitectonics (FENA) and by the NSF through grant DMS0402276 . 


\section{References}

1. G.S. Bales and D.C. Chrzan. Dynamics of irreversible island growth during submonolayer epitaxy. Phys. Rev. B, 50, 6057-6067, 1994.

2. W.K. Burton, N. Cabrera and F.C. Frank. The growth of crystals and the equilibrium structure of their surfaces. Phil. Trans. Roy. Soc. London Ser. A, 243, 299-358, 1951.

3. R.E. Caflisch, W.E., M.F. Gyure, B. Merriman, and C. Ratsch, Phys. Rev. E. 59, 6879 (1999).

4. R.E. Caflisch, Y.-J. Lee, S. Shu, Y. Xiao and Jinchao Xu. An application of multigrid methods for a discrete elastic model for epitaxial systems JCP, 219 (2006) 697-714.

5. S. Chen, M. Kang, B. Merriman, R.E. Caflisch, C. Ratsch, R. Fedkiw, M.F. Gyure and S. Osher. Level set method for thin film epitaxial growth. Journ. Comp. Phys., 167, 475-500, 2001.

6. D.J. Eaglesham and M. Cerullo, Phys. Rev. Lett. 64, 1943 (1990).

7. S. Guha, A. Madhukar, and K.C. Rajkumar, Appl. Phys. Lett. 57, 2110 (1990).

8. H.J. Kim, Z.M. Zhao, and Y.H. Xie, Phys. Rev. B 68, 205312 (2003).

9. L.D. Landau and E.M. Lifshitz, Theory of Elasticity, Butterworth-Heinemann, Oxford, UK, 1986.

10. R.E. Sunmi Lee, Caflisch and Y.-J. Lee Artificial Boundary Conditions for Discrete Elasticity SIAM J. Applied Math. 66 (2006) 1749-1775.

11. B. Li and R.E. Caflisch. Analysis of island dynamics in epitaxial growth. Multiscale Model. Sim., 1, 150-171, 2002.

12. Y.-W. Mo, D.E. Savage, B.S. Swartzentruber and M.G. Lagally, Phys. Rev. Lett. 65, 1020 (1990).

13. X. Niu, R. Vardavas, R.E. Caflisch and C. Ratsch. A Level Set Simulation of Directed Self-Assembly during Epitaxial Growth. Phys. Rev. B (2006) to appear.

14. E. Penev, P. Kratzer and M. Scheffler, Phys. Rev. B 64, 085401 (2001).

15. C. Ratsch, A.P. Seitsonen and M. Scheffler, Phys. Rev. B 55, 6750 (1997).

16. C. Ratsch, M.F. Gyure, S. Chen, M. Kang and D.D. Vvedensky. Fluctuations and scaling in aggregation phenomena. Phys. Rev. B, 61, 10598-10601, 2000.

17. C. Ratsch, M.F. Gyure, R.E. Caflisch, F. Gibou, M. Petersen, M. Kang, J. Garcia and D.D. Vvedensky. Level-set method for island dynamics in epitaxial growth. Phys. Rev. B, 65, \#195403, U697-U709, 2002.

18. A.E. Romanov, P.M. Petroff and J.S. Speck, Appl. Phys. Lett. 74, 2280 (1999).

19. A.C. Schindler, M.F. Gyure, D.D. Vvedensky, R.E. Caflisch, C. Connell and G. D. Simms. Theory of Strain Relaxation in Heteroepitaxial Systems Phys. Rev. B 67 (2003): art. no. 075316 